\title{
MICROCIRCULATORY DISORDERS IN WOMEN WITH POLYCYSTIC OVARY SYNDROME
}

\author{
Daniela Koleva, ${ }^{1}$ Mariana Batzelova, ${ }^{2}$ Julia Nikolova, ${ }^{3}$ Maria Orbetzova ${ }^{4}$
}

\begin{abstract}
It is believed that microvascular dysfunction plays a major role in the development of insulin resistance (IR). Performing nailfold capillaroscopy with evaluation of microvascular parameters in women with polycystic ovary syndrome (PCOS) would undoubtedly be helpful for the investigation of novel pathophysiological mechanisms in IR occurrence. The aim of the present study was to compare the values of microvascular parameters between PCOS patients and clinically healthy women and to assess its relationship with clinical and metabolic parameters in the PCOS women. Our study included 21 PCOS patients and 22 clinically healthy women (controls). Nailfold capillaroscopy was conducted and the following parameters were assessed: arterial limb diameter ( $\mathrm{d}$ art), venous limb diameter ( $\mathrm{d}$ ven), top diameter of loops (top d), length of loops, $\mathrm{d}$ art/d ven ratio, $\mathrm{d}$ ven/d art ratio, number of capillary loops (n cap), number of abnormal loops (\% abn cap) and a presence of perivascular diapedesis. Weight, height, fasting plasma glucose (FPG), immunoreactive insulin (IRI), lipid parameters, total testosterone, systolic (SBP) and diastolic blood pressure (DBP) were measured in the PCOS women. Body mass index (BMI) and homeostasis model of insulin resistance index (HOMA-IR) were calculated. We found significantly higher values of age, BMI and \% abn cap in the women with PCOS as compared to the controls. Interestingly, \% abn cap showed a negative correlation with age. Furthermore, an inverse association between top $d$ and the values of SBP and DBP was established. We determined a positive correlation between the presence of perivascular diapedesis and the values of FPG, HOMA-IR and testosterone. As a conclusion, the higher \% abn cap in the PCOS women might be an early pathophysiological sign of microvascular dysfunction. The positive correlation between perivascular diapedesis and the degree of IR, hyperglycemia, and hyperandrogenemia shows the existence of a chronic low-grade inflammatory process in PCOS.
\end{abstract}

UDC Classification: 616.89; DOI: http://dx.doi.org/10.12955/cbup.v5.1051

Keywords: capillaroscopy, microvascular dysfunction, insulin resistance

\section{Introduction}

Polycystic ovary syndrome (PCOS) is a condition that represents a specific prototype of metabolic syndrome (MS) among women at reproductive age. Patients with PCOS are characterized by typical clinical features of hyperandrogenemia and their metabolic status often includes android type of obesity, glucose tolerance disturbances, atherogenic dyslipidemia, and hypertension. Several studies have shown that PCOS women have a greater risk of developing type 2 diabetes mellitus (TDM2) as well as a low-grade chronic inflammatory process leading to endothelial dysfunction and coagulation and/or fibrinolysis abnormalities (Teede et al., 2010; Koleva et al., 2016).

It is known that microcirculation consists of terminal arterioles, capillaries, and venules. The main functions of the microcirculation are: 1) to accomplish the exchange of nutrients, oxygen, and hormones between plasma and tissue fluid; 2) to minimize the fluctuations in the hydrostatic pressure at the level of capillaries; 3) to adjust the peripheral vascular resistance, respectively the blood pressure (Muris et al., 2013). Certainly, the maintenance of normal microvascular function is important for the regulation of tissue homeostasis and arterial blood pressure (Levy et al., 2001). It has been found that microvascular dysfunction might cause disrupted glucose tissue utilization (Clark et al., 2003; Serne et al., 2001; Wallis et al., 2002) and peripheral vascular resistance (Antonios et al., 1999), resulting in the development of IR and arterial hypertension (AH).

It is believed that microvascular dysfunction might be a possible cause for the occurrence of metabolic disorders in individuals with obesity and insulin resistance syndromes. Undoubtedly, there is a deficiency of data concerning microcirculatory characteristics in women with PCOS. This was the reason for us to conduct a study which aimed to compare the values of microvascular parameters between women with PCOS (a representative model of IR syndrome) and clinically healthy women and to assess its relationship with clinical and metabolic parameters in the PCOS women.

\footnotetext{
${ }^{1}$ St. George" University Hospital, Clinic of Endocrinology and metabolic diseases, nelka_medicine@abv.bg

${ }^{2}$ Medical University of Plovdiv, Bulgaria, marali@abv.bg

${ }^{3}$ Medical University of Plovdiv, Bulgaria, junikol@yahoo.com

${ }^{4}$ St. George“ University Hospital, Clinic of Endocrinology and metabolic diseases, morbetzova@abv.bg
} 


\section{Materials and methods}

The present study was conducted in the Clinic of Endocrinology and Metabolic Diseases at "St. George" University Hospital, Plovdiv. It comprised of 21 young PCOS women (mean age $25.7 \pm 4.08$ years and mean BMI $30.78 \pm 8.08 \mathrm{~kg} / \mathrm{m}^{2}$ ) and 22 clinically healthy women (mean age of $21.9 \pm 4.10$ years and mean BMI $23.9 \pm 6.10 \mathrm{~kg} / \mathrm{m}^{2}$ ), serving as a control group.

Nailfold capillaroscopy using capillaroscope model JH1005, PRC was conducted in the Department of Physiology at Medical University of Plovdiv. Each woman was examined after 20 minutes rest in a room with normal temperature $\left(20-22^{\circ} \mathrm{C}\right)$. Visualization of the capillaries was realized after placing a drop of cedar oil on the nailfold of the fourth finger of the patient's both hands. The following microvascular parameters were investigated: arterial limb diameter $(\mathrm{d}$ art $) \mu \mathrm{m}$, venous limb diameter (d ven) $\mu \mathrm{m}$, top diameter of loops (top d) $\mu \mathrm{m}$, length of loops $\mu \mathrm{m}, \mathrm{d}$ art/d ven ratio, $\mathrm{d}$ ven/d art ratio, number of capillary loops (n cap), number of abnormal loops (\% abn cap) and a presence of perivascular diapedesis.

In all the PCOS women the following measurements and laboratory tests were performed: weight, height, fasting plasma glucose (FPG), fasting immunoreactive insulin (IRI), total cholesterol (TC), HDL-cholesterol (HDL-C), triglycerides (TG), total testosterone, systolic (SBP) and diastolic blood pressure (DBP). Venous blood samples were taken after a 12-hour overnight fast and were sent to Central Clinic Laboratory at "St. George "University Hospital.

Insulin was tested using a commercial kit for quantitative determination of immunoreactive insulin on the basis of microparticular immunoenzyme analysis (MEIA) on an AxSYM system (ABBOTT, USA) with the following characteristics: sensitivity $\leq 0.8 \mathrm{mIU} / \mathrm{ml}$; inter-assay variation, $\mathrm{CV} \%<2.9$; intraassay variation $\mathrm{CV} \%<5.3$. Serum glucose levels were determined by a standard GOD-POD method. TC was determined by ChOD, PAP; TG by GPO, PAP, and HDL-C by MgSO4-dextran SO4 precipitation, Schneiders Analysers; Netherlands test; Delta Kone Autoanalyzer.

Systemic blood pressure (SBP and DBP) was measured using a sphygmomanometer.

The diagnosis of PCOS was made according to the Rotterdam criteria (Rotterdam ESHRE/ASRMSponsored 2003), when the two of the following three features were present: oligo- and/or anovulation, clinical and/or biochemical signs of hyperandrogenism, and polycystic ovaries on ultrasound examination (the presence of $\geq 12$ follicles measuring 2-9 $\mathrm{mm}$ in diameter and/or ovarian volume $>10 \mathrm{~cm}^{3}$ ).

The statistical analysis was performed by SPSS version 21.0 for Windows. A comparative analysis of clinical, antropomethric and microcirculatory parameters was performed among the studied groups of women. The results are presented as mean \pm SD. Pearson ( $r$ ) and Spearman (rho) correlation coefficients for assessing correlations among the parameters were used.

\section{Results}

Table 1 presents clinical, anthropometric and microcirculatory characteristics in the studied groups of women.

Interestingly, $\%$ abn cap showed a negative correlation with age $(\mathrm{r}=-0.567, \mathrm{P}=0.043)$. Furthermore, an inverse association between top $\mathrm{d}$ and the values of SBP $(\mathrm{r}=-0.610, \mathrm{P}=0.017)$ and $\mathrm{DBP}(\mathrm{r}=-0.581$, $\mathrm{P}=0.037$ ) was established.

We determined a positive correlation between the presence of perivascular diapedesis and the values of FPG, HOMA-IR and testosterone (Table 2).

\section{Discussion}

Our results showed a higher percentage of abnormal capillary loops (elongated capillaries, increased tortuosity) in PCOS women compared to that in the clinically healthy women. A presence of perivascular diapedesis was found in $22.2 \%$ of the PCOS women. A positive correlation between perivascular diapedesis and the values of FPG, HOMA-IR, and testosterone was determined.

Leukocyte extravasation, also known as diapedesis, is the process of leukocytes movement out of the circulatory system towards the locus of tissue damage or inflammation. Leukocyte extravasation is most commonly observed in post-capillary venules, where haemodynamic shear forces are minimized. This process consists of several steps, namely: 1. chemoattraction; 2 . rolling adhesion; 3 . tight adhesion and 4. endothelial transmigration. Apart from an acute inflammatory disease, leukocyte 
extravasation might be perceived as a sign of chronic low-grade inflammation (Anderson and Anderson, 1976).

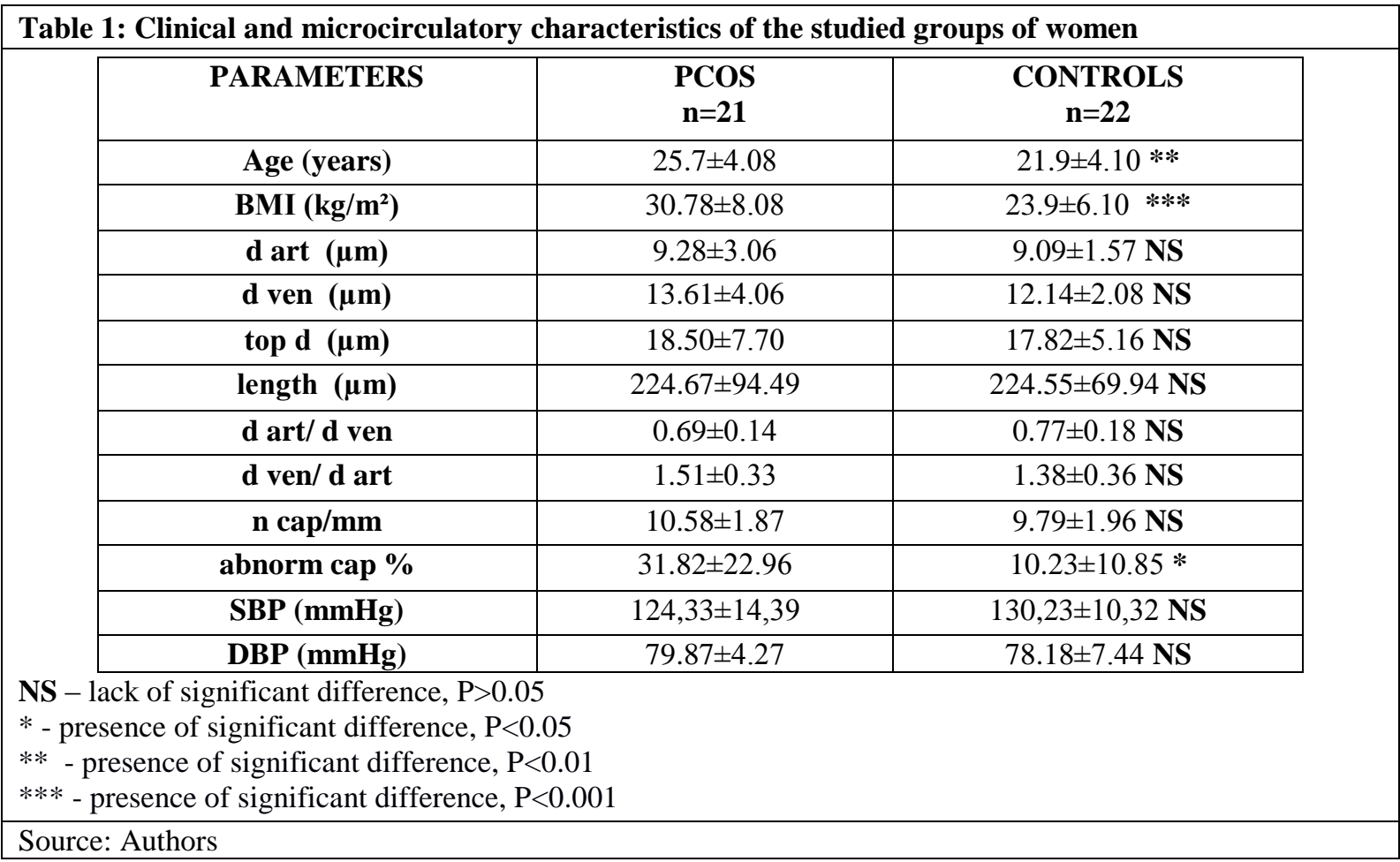

Table 2: Correlations (rho) between the presence of perivascular diapedesis and the values of FPG, HOMA-IR and total testosterone

\begin{tabular}{|c|c|c|}
\hline PARAMETERS & $\begin{array}{c}\text { Perivascular diapedesis } \\
\text { rho }\end{array}$ & P \\
\hline FPG $(\mathbf{m m o l} / \mathbf{l})$ & 0.588 & $\mathrm{P}=0.027$ \\
\hline HOMA-IR & 0.581 & $\mathrm{P}=0.029$ \\
\hline TESTOSTERONE $(\mathbf{n g} / \mathbf{m l})$ & 0.578 & $\mathrm{P}=0.039$ \\
\hline
\end{tabular}

Source: Authors

Our results showed the existence of an inverse relationship between the top $d$ and the values of SBP and DBP. This correlation may explain the pathophysiological mechanism of the development of arterial hypertension.

In general, arterial hypertension is characterized by both functional and structural microvascular changes. First, the mechanisms regulating vasomotor tone are considered to be abnormal, leading to enhanced vasoconstriction or reduced vasodilatation. Second, decreases in arteriolar diameters and increases in the wall-to-lumen ratio of small arteries have been demonstrated (Levy et al., 2001; Houben et al., 1995). Furthermore, a reduction in the density (rarefaction) of arterioles, venules, and capillaries can be observed in different vascular beds (Houben et al., 2005).

Endothelial dysfunction in hypertensive patients might be explained by the presence of suppressed vasodilator response and altered capillary activation under the influence of endothelium-dependent typical vasodilators (acetylcholine) as well as shear stress itself (Serne et al., 2001). Recently, several researchers in this field have described the occurrence of a disrupted insulin-mediated NO-dependent vasodilation in various models of hypertensive animals (Debbabi et al., 2006; Eringa et al., 2004). In addition, hypertension is characterized by a parallel increase in the production of the biological constrictive agents such as endothelin-1 (ET-1) and angiotensin II (Ang II) (Li et al., 2010).

Microvascular dysfunction may be secondary - due to permanently increased levels of blood pressure (Serne et al., 2002), as well as primary - representing a cause, not a consequence of hypertension. Microvascular disturbances may occur at a very early stage in the development of hypertension. Changes in the capillaries (similar to those described in the already established hypertension) can also 
be observed in patients with prehypertension or in normotensive individuals with a familial predisposition to the development of hypertension (de Jongh et al., 2004).

Pazos-Moura et al. (1990) conducted a study including 15 healthy individuals and 16 patients with TDM2 (a model of insulin resistance syndrome). Direct intravital microscopic examination of nailfold capillaries was performed in all the studied subjects. The results showed an increased number of enlarged capillaries in the TDM2 patients as compared to the controls. Besides, capillaries with nodular apical elongations were found only in the TDM2 group. In addition, capillary blood flow velocity (CBFV) was measured during rest and after the release of $60 \mathrm{~s}$ arterial occlusion. In order to assess the autoregulatory capacity the investigators determined peak CBFV after occlusion and time to reach it in single capillaries. The values of mean resting CBFV were not statistically different in the two groups but mean peak CBFV post occlusion was found to be significantly lower (controls: $1.49 \pm 0.14 \mathrm{~mm} / \mathrm{s}$; T2DM: $0.93 \pm 0.13 \mathrm{~mm} / \mathrm{s}, \mathrm{P}<0.05$ ) and mean time to reach it significantly prolonged (controls: $8.9 \pm 0.6 \mathrm{~s}$; T2DM: $18.0 \pm 1.9 \mathrm{~s} ; \mathrm{P}<0.05$ ) in the diabetics compared to the controls.

In a study of Kraemer-Aguiar et al (2008) 36 subjects with MS (National Cholesterol Education Program-Adult Treatment Panel III criteria) (10 men/26 women, $38.8 \pm 7.9$ years, $35.8 \pm 4.9 \mathrm{~kg} / \mathrm{m}^{2}$ ) with normal glucose tolerance (American Diabetes Association criteria) and 16 controls (11 men/5 women, $33.6 \pm 8.4$ years, $23.9 \pm 3.6 \mathrm{~kg} / \mathrm{m}^{2}$ ) were studied using nailfold videocapillaroscopy. Afferent, efferent, and apical capillary diameters; functional capillary density; red blood cell velocity (RBCV) at baseline; and $\mathrm{RBCV}(\max )$ and time $(\mathrm{TRBCV}(\max ))$ to reach it during postocclusive reactive hyperemia after 1-minute arterial occlusion were measured. Subjects with MS had smaller afferent, efferent, and apical diameters (4.2 [3.8-4.2] vs 5.6 [4.65-6.25] mum, $\mathrm{P}<0.001 ; 4.8$ [4.2-4.8] vs 6.2 [5.6-7] mum, $\mathrm{P}<0.001$; and 5.2 [4.8-5.55] vs 7.4 [6.2-8] mum, $\mathrm{P}<0.001)$; lower functional capillary density (7.28 [6.37-9.10] vs 10.4 [9.1-11.8] capillaries per square millimeter, $\mathrm{P}<0.001)$, RBCV $(0.62$ [0.57-0.65] vs 0.79 [0.76-0.89] mm/s, $\mathrm{P}<0.001]$, and $\mathrm{RBCV}(\max )(1.14$ [1.12-1.210] vs 1.57 [1.45$1.62] \mathrm{mm} / \mathrm{s}, \mathrm{P}<0.001)$; and longer TRBCV(max) (10.0 [10-11] vs 4.5 [4-6] s, $\mathrm{P}<0.001)$ compared with controls. Tha data show that microcirculatory dysfunction was associated with BMI.

A study of Lakhani et al. (2005) aimed to assess microvascular function in 12 PCOS women and 12 age-matched controls. The investigators conducted observation of forearm skin microvascular erythrocyte flux responses to cumulative doses of $1 \%$ acetylcholine (Ach) and $1 \%$ sodium nitroprusside (SNP), using laser Doppler imaging. Basal microvascular perfusion was found to be comparable in the two groups of women. However, the increase of skin microvascular perfusion in response to Ach was blunted in the PCOS women $(\mathrm{P}=0.018)$. Furthermore, peak Ach-induced erythrocyte flux was less in the PCOS patients $(125.1 \pm 21.7)$ than in the controls $(200.8 \pm 28.5)$. Additional covariance analysis determined that the aforementioned effect was unrelated to differences in BMI or serum testosterone, but IRI might be a weak confounder. As far as the response to SNP was concerned, no differences were found between the PCOS and the control groups. The authors concluded that the study was the first demonstration of the presence of microvascular endothelial dysfunction (an inhibited vasodilatory response to Ach) in women with PCOS.

Hyperandrogenemia is a typical endocrine feature of PCOS. It had been proved that testosterone could influence vasocontractile responses impairing endothelium-dependent relaxation (Adams et al., 1995; Hutchinson et al., 1997) in hypercholesterolaemic rabbits and monkeys. However, more recent studies have suggested that the vascular effects of testosterone might be more complex. It was found that acute exposure even to low doses of testosterone might significantly potentiate endothelin-1-induced vasoconstriction in porcine coronary artery rings (Teoh et al., 2000). This effect was insensitive to flutamide (an androgen receptor antagonist) and was not blocked by de novo protein synthesis inhibitors, suggesting that it was not mediated via the classical androgen receptor influence on gene transcription. Nevertheless, this fact does not detract from the possibility that the diminished AChinduced microvascular perfusion response seen in the aforementioned study of Lakhani et al. (2005) resulted, at least in part, from the elevated levels of androgens in women with PCOS. ANCOVA was used to test the effect of testosterone on the difference in ACh-induced erythrocyte flux between the control and PCOS groups. The testosterone-adjusted erythrocyte flux difference remained significant, suggesting that the effect of PCOS on ACh-induced microvascular blood flow was not related to differences in serum testosterone between the controls and the PCOS women. 
We may suggest that microvascular dysfunction in women with PCOS can be explained primarily with the accompanying obesity, not with the presence of hyperandrogenemia in PCOS, which was confirmed by the study of Ketel et al. (2008).

\section{Conclusion}

The higher \% abn cap in the PCOS women might be an early pathophysiological sign of microvascular dysfunction. The positive correlation between perivascular diapedesis and the degree of IR, hyperglycemia, and hyperandrogenemia shows the existence of a chronic low-grade inflammatory process in PCOS.

\section{References}

A. (2001). Capillary recruitment is impaired in essential hypertension and relates to insulin's metabolic and vascular actions. Cardiovasc Res, 49, 161-168.

A. (2002). Direct evidence for insulin-induced capillary recruitment in skin of healthy subjects during physiological hyperinsulinemia. Diabetes, 51, 1515-1522.

Adams, M.R., Williams, J.K. and Kaplan, J.R. (1995). Effects of androgens on coronary artery atherosclerosis and atherosclerosis-related impairment of vascular responsiveness. Arterioscler Thromb Vasc Biol 1995, 15, 562-570.

Anderson, A.O., Anderson, N.D. (1976). Lymphocyte emigration from high endothelial venules in rat lymph nodes. Immunology, 31(5), 731-748.

Antonios, T.F., Singer, D.R., Markandu, N.D., Mortimer P.S., MacGregor G.A. (1999). Rarefaction of skin capillaries in borderline essential hypertension suggests an early structural abnormality. Hypertension, 34, 655-558.

Clark, M.G., Wallis, M.G., Barrett, E.J., Vincent M.A., Richards S.M., Clerk L.H., Rattigan S. (2003). Blood flow and muscle metabolism: a focus on insulin action. Am J Physiol Endocrinol Metab, 284, E241-258.

de Jongh, R.T., Serne, E.H., Ijzerman, R.G., de Vries G., Stehouwer C.D. (2004). Impaired microvascular function in obesity: implications for obesity-associated microangiopathy, hypertension, and insulin resistance. Circulation, 109, $2529-2535$.

Debbabi, H., Uzan, L., Mourad, J.J., Safar M., Levy B.I., Tibiriçà E. (2006). Increased skin capillary density in treated essential hypertensive patients. Am J Hypertens, 19, 477-83.

Eringa, E.C., Stehouwer, C.D., van Nieuw Amerongen, G.P., Ouwehand L., Westerhof N., Sipkema P. (2004). Vasoconstrictor effects of insulin in skeletal muscle arterioles are mediated by ERK1/2 activation in endothelium. Am J Physiol Heart Circ Physiol, 287, H2043-2048.

Houben, A.J., Canoy, M.C., Paling, H.A., Derhaag, P.J., de Leeuw, P.W. (1995). Quantitative analysis of retinal vascular changes in essential and renovascular hypertension. J Hypertens, 13, 1729-1733.

Houben, A.J., Willemsen, R.T., van de Ven, H., de Leeuw, P.W. (2005). Microvascular adaptation to changes in dietary sodium is disturbed in patients with essential hypertension. J Hypertens, 23, 127-132.

Hutchinson, S.J., Sudhir, K., Chou, T.M., Sievers R.E., Zhu B.Q., Sun Y.P., Deedwania P.C., Glantz S.A., Parmley W.W., Chatterjee K. (1997). Testosterone worsens endothelial dysfuction associated with hypercholesterolemia and environmental tobacco smoke exposure in male rabbit aorta. J Am Coll Cardiol, 29, 800-807.

Ketel, I.J., Stehouwer, C.D., Serne, E.H., Korsen T.J., Hompes P.G., Smulders Y.M., de Jongh R.T., Homburg R., Lambalk C.B. (2008) Obese but not normal-weight women with polycystic ovary syndrome are characterized by metabolic and microvascular insulin resistance. J Clin Endocrinol Metab, 93, 3365-72.

Koleva, D.I., Orbetzova M.M., Nikolova J.G., Tyutyundzhiev S.B. (2016). Adipokines and soluble cell adhesion molecules in insulin resistant and non-insulin resistant women with polycystic ovary syndrome, Archives of Physiology and Biochemistry, 122(4):223-227.

Kraemer-Aguiar, L.G., Laflor, C.M., Bouskela, E. (2008). Skin microcirculatory dysfunction is already present in normoglycemic subjects with metabolic syndrome. Metabolism Clinical and Experimental, 57, 1740 -1746.

Lakhani, K., Leonard, A., Seifalian, A.M., Hardiman P. (2005). Microvascular dysfunction in women with polycystic ovary syndrome. Human Reproduction, 20(11), 3219-3224.

Levy, B.I., Ambrosio, G., Pries, A.R, Struijker-Boudier H.A. (2001). Microcirculation in hypertension: a new target for treatment? Circulation, 104, 735-740.

Li, R., Zhang, H., Wang, W., Wang X., Huang Y., Huang C., Gao F. (2010). Vascular insulin resistance in prehypertensive rats: role of PI3-kinase/Akt/eNOS signaling. Eur J Pharmacol, 628, 140-147.

Pazos-Moura, C.C., Moura, E.G., Bouskela, E., Torres Filho I.P., Breitenbach M.M. (1990) Nailfold capillaroscopy in noninsulin dependent diabetes mellitus: blood flow velocity during rest and post-occlusive reactive hyperaemia. Clin Physiol, 10(5), 451-461.

Serne, E.H., Gans, R.O., Nijveldt, R., Stehouwer C.D.Serne, E.H., Gans, R.O., ter Maaten, J.C., ter Wee P.M., M Donker A.J., Stehouwer C.D.Teede, H., Deeks, A., and Moran, L. (2010). Polycystic ovary syndrome: a complex condition with psychological, reproductive and metabolic manifestations that impacts on health across the lifespan. BMC Medicine, 8, 41 .

Teoh, H., Quan, A. and Man, R.Y. (2000). Acute impairment of relaxation by low levels of testosterone in porcine coronary arteries. Cardiovasc Res, 45, 1010-1018.

Wallis, M.G., Wheatley, C.M., Rattigan, S., Barrett E.J, Clark A.D., Clark M.G.. (2002). Insulin-mediated hemodynamic changes are impaired in muscle of Zucker obese rats. Diabetes, 51, 3492-3498. 